\title{
A Spectrum of Soft Computing Model for Medical Diagnosis
}

\author{
Pankaj Srivastava* and Neeraja Sharma \\ Department of Mathematics, Motilal Nehru National Institute of Technology, Allahabad, Uttar Pradesh, 211004, India
}

Received: 7 Jun. 2013, Revised: 12 Oct. 2013, Accepted: 13 Oct. 2013

Published online: 1 May. 2014

\begin{abstract}
The present paper is focused on design and development of soft computing decision making model to solve real life complex problems related with medical sciences.
\end{abstract}

Keywords: Linguistic Strings, Rule base, Degree of Match

\section{Introduction}

It is well known fact that decision making processes in real life problems are too complex. The interplay of impreciseness, vague features makes situation too critical especially in medical science where medical experts take decision on their experience rather than follow guideline of any scientific model designed. The day-today practices of medical practitioners enhance their knowledge base but it is difficult to retain and apply the entire knowledge base by them in every medical case. In medical diagnosis, where the diagnosis is totally based on humans abilities involving their intuition, and a number of uncertain factors affecting the body of the patient, soft computing mechanisms are very useful and effective. The soft computing process deals effectively in medical diagnosis as it squeezes knowledge base of the experts and design guideline on the basis of framed rules that match experts observation. The key tool of soft computing technology is fuzzy tools as they provide a scientific approach to measure vague, imprecise inadequate expertises and ambiguous medical data. The decision making process in the presence of fuzzy variables presented by Jain, Ramesh[5] and later on Zadeh, L.A. and Bellman, R.E.[1] proposed application of fuzzy tools in the field of medicine. Cho, Seongwon, Ersoy, Okan K and Lehto, Mark [3]designed an algorithm which described for the computation of the degree of match (DM) between the antecedent part of a classification rule and an assertion given by a user when imprecise and precise propositions are mixed freely in a fuzzy expert classification system,
Degani, Rosanna[4] investigated the computerized analysis of electrocardiographic signals by a model where the imprecise knowledge of the state of cardiac system and the vague idea of the pathological classes are taken care of by means of the fuzzy set formalism. Jena, R. K. et al[6] threw light on soft computing paradigm in bioinformatics with particular emphasis on integrative research. Pandey, D et al[8] gave a fuzzy analysis of ECG diagnosis using the principles of fuzzy logic for cardiac analysis with the knowledge of medical experts, like physicians and the cardiologists, and established the rule based system, Verma, Priyanka[14] proposed an approach to study classification scheme of ECG beats on the basis of soft computing methods. Begum, Shahin Ara and Devi, O. Mema[2] surveyed the algorithmic methods of fuzzy pattern recognition for medical imaging. Recently, Srivastava, Pankaj and Srivastava, Amit[11, 12] designed a fuzzy expert system to determine coronary heart disease (CHD) risk of patients in India and assessed person's risk as well as recommended whether person has to live life normally or with diet or with drug treatment, they also worked to design a user friendly, intelligent and effective diagnosis system for risk assessment of hypertension respectively. Srivastava, Pankaj, Srivastava, Amit and and Sirohi, Ritu [13] proposed soft computing classification criterion to design and develop user friendly diagnostic system for Hepatitis B. Srivastava, Pankaj and Sharma, Neeraja[9] developed soft computing criterion for classification of ECGs beats which are responsible for various phases of cardiac related problems and these phases have been categorised under the fuzzy tools on the

\footnotetext{
*Corresponding author e-mail: drpankaj23@gmail.com
} 
available ECG beats further Srivastava, Pankaj, Sharma, Neeraja and Singh, Richa[10] proposed soft computing diagnostic system for diabetes that will sharpen the diagnostic process as well as guide patients to evolve strategies to control their sugar level.

In the present article we proposed a soft computing model to deal medical diagnosis process effectively. The proposed model is tested in the case of ECG analysis and the satisfactory factor measured with Degree of Match criterion under the domain of considered inputs and computed output.

\section{Preliminaries}

The following features of fuzzy have been considered in designing the model.

\subsection{Definitions}

\section{(I) Fuzzy Set}

Let $\mathrm{U}$ be a non-empty set called universe of discourse or simply domain. Then a fuzzy set A on $\mathrm{U}$ is expressed by a membership function $\mu_{A}: U \rightarrow[0,1], \mu_{A}(x)$ is called membership grade of $\mathrm{x}$ in $\mathrm{A}$ :

$$
\mathrm{A}=\left\{\left(x, \mu_{A}(x)\right): x \in U\right\}
$$

\section{(II) Intersection of Two Fuzzy Sets}

Let $A$ and $B$ be two fuzzy sets in universe of discourse $\mathrm{U}$ and their membership functions are $\mu_{A}$ and $\mu_{B}$. Then, fuzzy intersection $\cap$ on $A$ and $B$ or AND operation (AND operation is used in fuzzy logic) such as:

$$
\mathrm{A} \cap \mathrm{B}=\left\{\left(x, \min \left(\mu_{A}(x), \mu_{B}(x)\right)\right): x \in U\right\}
$$

\section{(III) Linguistic Terms}

In traditional mathematics variable is usually a numeric number where in fuzzy logic application linguistic terms are those variables whose values are not numbers but words or sentences which express concepts and knowledge in human language.

\section{(IV) Fuzzy If-Then Rule}

In fuzzy inference system, fuzzy if-then rule is a scheme for capturing imprecise and uncertain knowledge. A fuzzy if-then rule associates a condition using suitable AND/OR operation in described linguistic variables and fuzzy sets to a conclusion.

\section{(V) Degree of Match}

The degree of match (DM) measures the satisfactory factor between inputs and outputs. It is calculated using the grade of membership value of factor (input/ output) in its corresponding fuzzy set.

\section{Decision Making Method}

The soft computing model functions under the proposed algorithm which is as follows:

\subsection{Algorithm}

[1] Firstly imprecise and vague facts are categorised in 'r' input fuzzy sets $X_{i}, \mathrm{i}=1,2, \ldots, \mathrm{r}$, and 'n' outputs $B_{t}$, $\mathrm{t}=1,2, \ldots, \mathrm{n}$, as then respective possibility.

[2] Divide each fuzzy set in $k_{i}$ linguistic terms $L_{i j}$ for $\mathrm{i}=1,2, \ldots, \mathrm{r}$ and $\mathrm{j}=1,2, \ldots, k_{i}$.

[3] Construct suitable membership function for each linguistic term in each fuzzy set using the available data.

[4] Develop $\mathrm{m}=k_{1} k_{2} \ldots k_{r}$ linguistic strings $J_{K}, \mathrm{~K}=1$, $2, \ldots, \mathrm{m}$ using AND operation on each linguistic term $L_{i j}$ taken from each fuzzy set $X_{i}$ for $\mathrm{i}=1,2, \ldots, \mathrm{r}$ and $\mathrm{j}=1,2, \ldots, k_{i}$.

[5] Represent the state of the system by a fuzzy set A in which elements are $\mathrm{m}$ linguistic strings and the value of each linguistic string is the minimum of all linguistic terms.

[6] Develop possible fuzzy if-then rules with the help of fuzzy expert system.

[7] Construct a utility matrix $\mathrm{U}$ of $\mathrm{m} \mathrm{x}$ n order between $\mathrm{m}$ linguistic strings and $\mathrm{n}$ alternatives based on designed fuzzy rules.

[8] Design $\mathrm{n}$ fuzzy utility sets $U_{I}, \quad \mathrm{I}=1, \quad 2, \ldots, \mathrm{n}$ corresponding to each alternative using $x \oplus y=\mathrm{x}+\mathrm{y}-$ xy for each $x, y \in U$.

[9] Develop $\mathrm{n}$ maximizing sets $U_{M I}, \mathrm{I}=1,2, \ldots, \mathrm{n}$ corresponding to each alternative.

[10] Develop n optimal fuzzy utility sets $U_{O I}, \mathrm{I}=1,2, \ldots, \mathrm{n}$. Each optimal fuzzy set $U_{O I}$ is obtained by fuzzy intersection $\wedge$ on fuzzy utility set and maximizing set such that $\mu_{U_{O I}}(x)=\mu_{U_{I}}(x) \cap \mu_{U_{M T}}(x)=\min \left(\mu_{U_{I}}(x)\right.$, $\mu_{U_{M I}}(x)$ ) for each utility value $\mathrm{x}$.

[11] Take maximum membership value from each optimal utility fuzzy set.

[12] The optimal alternative $B_{O}$ with corresponding maximum membership grades obtained in step 11 such as

$B_{O}=\left\{\left((\max ) \mu_{U_{O I}}(x), B_{I}\right): \forall \in U_{O I}\right\}$

for $\mathrm{I}=1,2, \ldots, \mathrm{n}$.

[13] To know what extent our result is satisfied by given inputs and computed outputs, the degree of match method is applied to know the satisfactory factor.

[14] Obtain values of degree of match $D M_{i}$ of input for $\mathrm{i}=1,2, \ldots, \mathrm{r}$ between given precise value of input $x_{i}$ and fuzzy input set $X_{i}$ given as $D M_{i}=2 \mu_{X_{i}}\left(x_{i}\right)-1$.

[15] Take the minimum from all degree of matches $D M_{i}$ for $\mathrm{i}=1,2, \ldots, \mathrm{r}$ which is denoted by total degree of match $D M_{T}$ of input

$D M_{T}=\min \left\{D M_{1}, D M_{2}, \ldots, D M_{r}\right\}$

Also find the degree of match $D M_{O}$ of optimal alternative.

[16] In order to measure satisfactory features, find the difference $\mathrm{D}=\left|D M_{T}-D M_{O}\right|$. If $0 \leq D<1$ or very close to zero, then the output is satisfied to fuzzy inputs.

[17] Develop Sugeno spikes of optimal alternatives. 
The flow chart of this algorithm is given in figure 1.

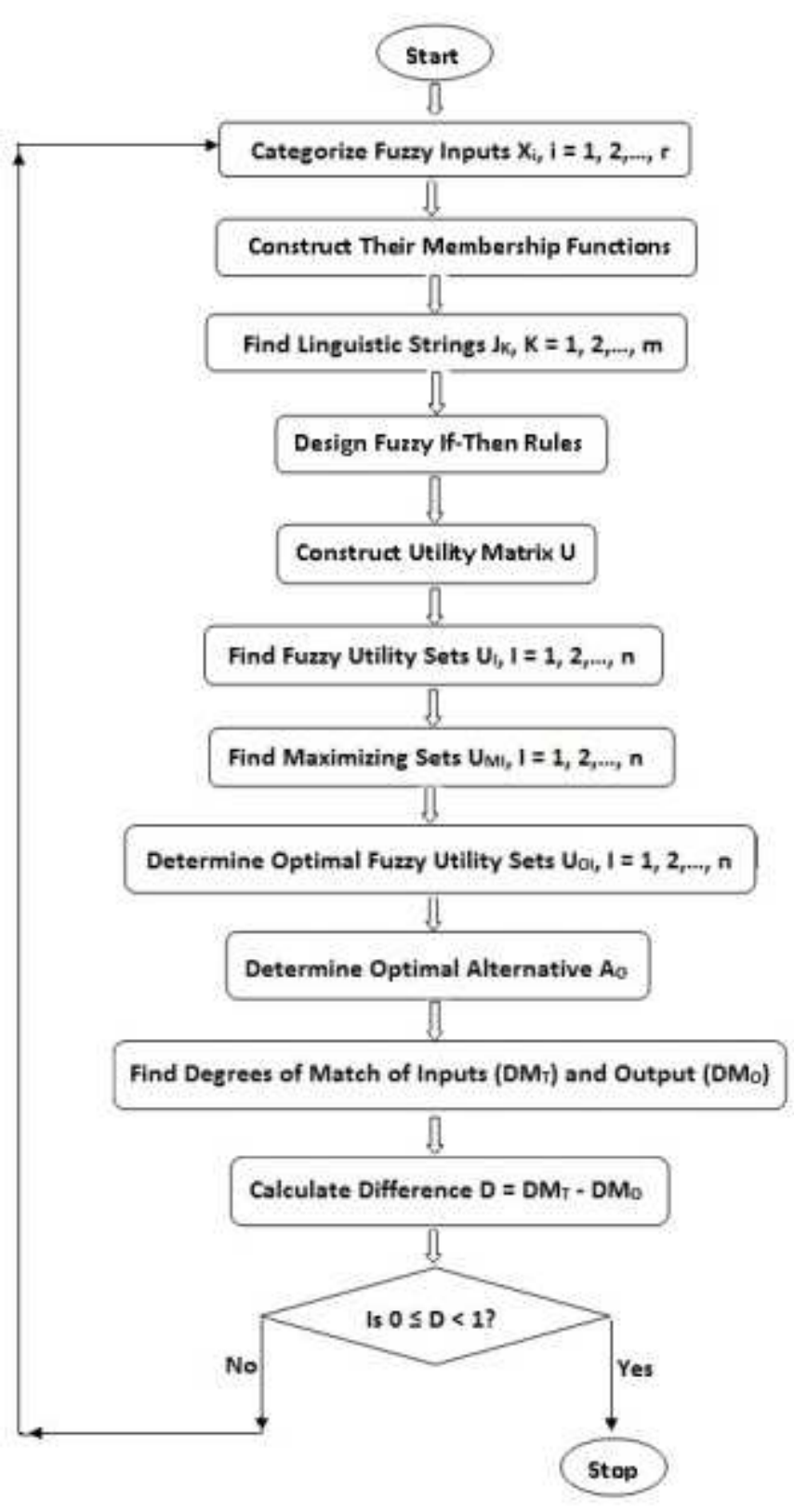

Fig. 1: Flow Chart

\section{Case Study}

We apply our model to examine health status of heart of the patient concerned, the basic feature of the concerned ECG graph are as follows:
$\mathrm{PR}$ duration $=0 \mathrm{sec}$

QR height $=4.5 \mathrm{~mm}$

$\mathrm{RS}$ height $=4.0 \mathrm{~mm}$

QRS duration $=0.078 \mathrm{sec}$

$\mathrm{R}-\mathrm{R}$ interval $=30 \mathrm{~mm}$

Here, we have taken two fuzzy variables namely, QRS angle and R-R interval with their membership functions given below:

(1) QRS angle is categorised in following eight linguistic variables with membership functions given below:

$\begin{array}{lc}\text { Negative very large (NVL) } & -150^{\circ} \text { to }-180^{\circ} \\ \text { Negative large (NL) } & -90^{\circ} \text { to }-150^{\circ} \\ \text { Negative medium (NM) } & -30^{\circ} \text { to }-90^{\circ} \\ \text { Negative small (NS) } & 0^{\circ} \text { to }-30^{\circ} \\ \text { Positive small (PS) } & 0^{\circ} \text { to } 30^{\circ} \\ \text { Positive medium (PM) } & 30^{\circ} \text { to } 110^{\circ} \\ \text { Positive large (PL) } & 110^{\circ} \text { to } 150^{\circ} \\ \text { Positive very large (PVL) } & 150^{\circ} \text { to } 180^{\circ}\end{array}$

$1 \quad \mu_{N V L}(x)=\exp ^{\left(((x+180) / 30)^{2}\right) /-2}$

$2 \mu_{N L}(x)=\exp ^{\left(((x+150) / 60)^{2}\right) /-2}$

$3 \quad \mu_{N M}(x)=\exp ^{\left(((x+90) / 60)^{2}\right) /-2}$

$4 \mu_{N S}(x)=\exp ^{\left(((x+30) / 30)^{2}\right) /-2}$

$5 \quad \mu_{P S}(x)=\exp ^{\left(((x-30) / 30)^{2}\right) /-2}$

$6 \mu_{P M}(x)=\exp ^{\left(((x-110) / 80)^{2}\right) /-2}$

$7 \quad \mu_{P L}(x)=\exp ^{\left(((x-150) / 40)^{2}\right) /-2}$

$8 \quad \mu_{P V L}(x)=\exp ^{\left(((x-180) / 30)^{2}\right) /-2}$

(2) R-R interval is categorised into three linguistic variables represented below with their membership functions and ranges:

$\begin{array}{ll}\text { Short } & <15 \mathrm{~mm} \\ \text { Normal } & 15-25 \mathrm{~mm} \\ \text { Large } & >25 \mathrm{~mm}\end{array}$

1. $\mu_{\text {short }}(x)= \begin{cases}1 & x \leq 10 \\ \frac{(15-x)}{5} & 10<x<15 \\ 0 & x \geq 15\end{cases}$

2. $\mu_{\text {normal }}(x)= \begin{cases}0 & x \leq 10 \text { or } x \geq 30 \\ \frac{(x-10)}{5} & 10<x<15 \\ 1, & 15 \leq x \leq 25 \\ \frac{(30-x)}{5} & 25<x<30\end{cases}$

$3 . \mu_{\text {large }}(x)= \begin{cases}0, & x \leq 25 \\ \frac{(x-25)}{5} & 25<x<30 \\ 1, & x \geq 30\end{cases}$

On the basis of eight linguistic variables of QRS angle and three linguistic variables of R-R interval we have 24 linguistic strings as follow:

1. $J_{1}=N V L_{Q R S} S_{R R}$

2.J $J_{2}=N V L_{Q R S} L_{R R}$

- ... 
$11 . J_{11}=N S_{Q R S} L_{R R}$

12.J $J_{12}=N S_{Q R S} N_{R R}$

$-\ldots$

23. $J_{23}=P V L_{Q R S} L_{R R}$

24.J $J_{24}=P V L_{Q R S} N_{R R}$

Now 48 fuzzy if-then rules constructed with the help of fuzzy expert system given below:

1.If Angle is NVL and R-R interval is short, then Health Status is rare.

2.If Angle is NVL or R-R interval is short, then Health Status is rare.

3.If Angle is NVL and R-R interval is large, then Health Status is rare.

4.If Angle is NVL or R-R interval is large, then Health Status is rare.

5.If Angle is NVL and R-R interval is normal, then Health Status is little bit normal.

$-\ldots$

21.If Angle is NS or R-R interval is normal, then Health Status is normal.

22.If Angle is NS and R-R interval is normal, then Health Status is normal.

23.If Angle is NS or R-R interval is large, then Health Status is little bit normal.

24.If Angle is NS and R-R interval is large, then Health Status is little bit normal.

25.If Angle is PS and R-R interval is normal, then Health Status is normal.

$-\ldots$

44.If Angle is PVL or R-R interval is normal, then Health Status is somewhat abnormal.

45.If Angle is PVL and R-R interval is large, then Health Status is abnormal.

46.If Angle is PVL or R-R interval is large, then Health Status is abnormal.

47.If Angle is PVL or R-R interval is short, then Health Status is abnormal.

48.If Angle is PVL and R-R interval is short, then Health Status is abnormal.

The utility matrix $U$ designed of order $5 \times 24$ as per rule base:

$$
\mathrm{U}=\left(\begin{array}{llllllll}
9 & 9 & \ldots & 6 & 1 & \ldots & 8 & 7 \\
6 & 6 & \ldots & 8 & 5 & \ldots & 4 & 3 \\
1 & 1 & \ldots & 5 & 9 & \ldots & 1 & 2 \\
4 & 4 & \ldots & 2 & 4 & \ldots & 8 & 7 \\
7 & 7 & \ldots & 3 & 1 & \ldots & 9 & 8
\end{array}\right)
$$

The given fuzzy set which represents the of state of the concerned patient:

$1 . \mathrm{QRS}$ angle $=\{(0.0019, \mathrm{NL}),(0.0393, \mathrm{NM}),(0.0085$, NS), (0.5531, PS), (0.8393, PM), (0.2453, PL $\}$

2.R-R interval $=\{(0, S),(1, L),(0, N)\}$

$\mathrm{A}=\left\{\left(0.019, J_{5}\right),\left(0.0393, J_{8}\right),\left(0.0085, J_{11}\right),(0.5531\right.$, $\left.\left.J_{14}\right),\left(0.8393, J_{17}\right),\left(0.2453, J_{20}\right)\right\}$
The health status of the heart is categorised in five outputs: $b_{1}=$ Rare, $b_{2}=$ Little bit normal, $b_{3}=$ Normal, $b_{4}$ $=$ Somewhat abnormal, $b_{5}=$ Abnormal

The required fuzzy utility sets are as follows:

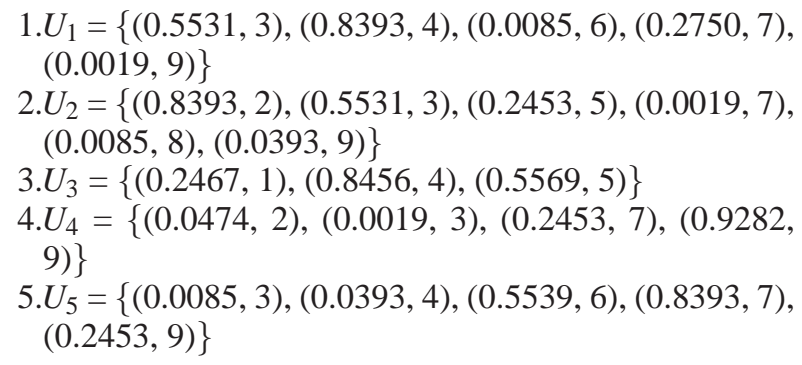

The maximizing sets corresponding to each alternatives are:

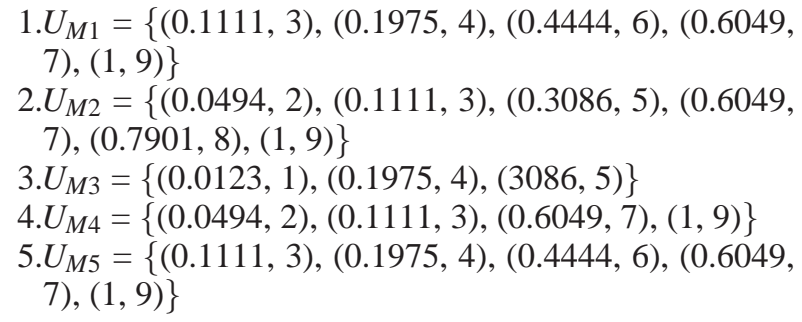

As per step [10] in algorithm, the optimal fuzzy utility sets are given below:

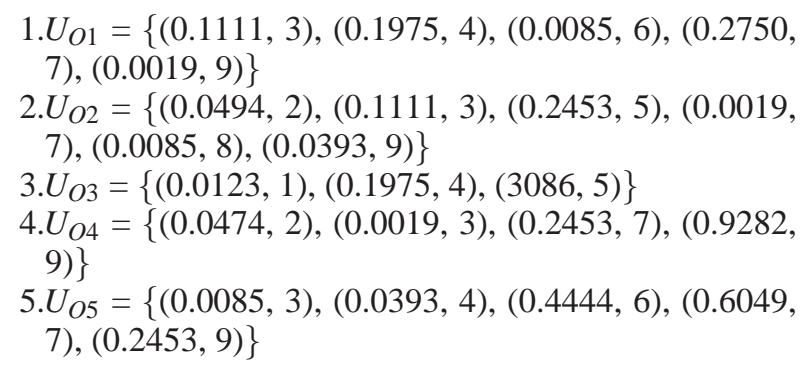

The optimal alternative as per step [12] as follows:

$B_{O}=\{(0.2750$, rare $),(0.2453$, little bit normal $)$, (0.3086, normal), (0.9282, somewhat abnormal), (0.6049, abnormal) $\}$ 


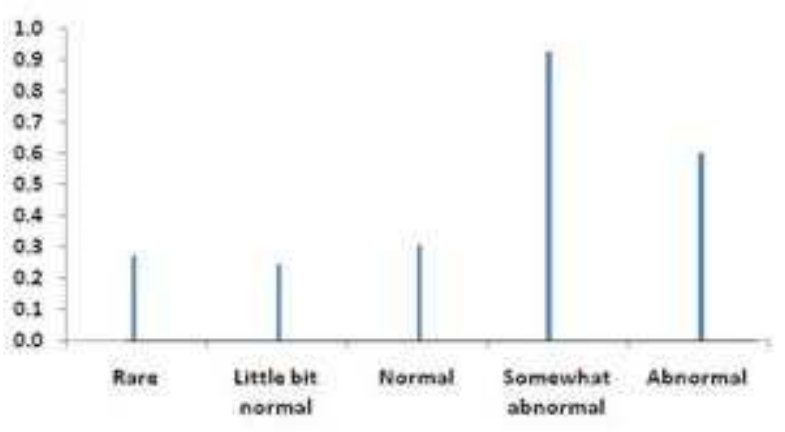

Fig. 2: Membership Functions of Optimal Alternative below:

Degrees of match for inputs as in step [14] as given

QRS angle is $62.6502^{\circ}$ and R-R interval is $30 \mathrm{~mm}$, so their degree of match are

$D M_{1}=2 \mu_{Q R S}(62.6502)-1=2(0.8393)-1=0.6786$

$D M_{2}=2 \mu_{R-R}(30)-1=2(1)-1=1$

Total degree of match of input $D M_{T}=\min \{0.6786,1\}$ $=0.6786$.

The degree of match of optimal alternative is $D M_{O}=$ $2(0.9282)-1=0.8564$

Difference $\mathrm{D}=\left|D M_{T}-D M_{O}\right|=|0.8564-0.6786|=$ 0.1778 , which lies between 0 and 1 and close to zero, that suggests the noise between input observations and output observations are approximately close to each other and verifies level of satisfaction.

\section{Conclusion}

The Satisfactory Criterion measured by the designed soft computing model has been verified by the medical expert and it can be extented in other domain as well.

\section{References}

[1] Bellman, R. E. and Zadeh, L. A., Decision making in a fuzzy environment, Management Science, 17, B141-B164 (1970).

[2] Begum, Shahin Ara and Devi, O. Mema, Fuzzy Algorithms for Pattern Recognition in Medical Diagnosis, Assam University Journal of Science and Technology, 7, 1-12 (2011).

[3] Cho, Seongwon, Ersoy, Okan K and Lehto, Mark, An Algorithm to Compute Degree of Match in Fuzzy Systems, Fuzzy Sets and Systems, 49, 285-299 (1992).

[4] Degani, Rosanna, Computerized Electrocardiogram Diagnosis: Fuzzy Approach, Methods of Information in Medicine, 31, 225-233 (1992).
[5] Jain, Ramesh, Decision making in the Presence of Fuzzy Variables,IEEE Transactions on Systems, Man, and Cybernetics, 6, 698-703 (1967).

[6] Jena, R. K and et.al., Soft Computing Methodologies in Bioinformatics, European Journal of Scienific Research, 26, 189-203 (2004).

[7] Klire, George. J. and Yuan, Bo. Fuzzy Sets and Fuzzy Logic: Theory and Applications, PHI Learning Private Limited: New Delhi, (2009).

[8] Pandey, D., Mahajan, Vashali and Srivastava, Pankaj, Rule Based System for Cardiac Analysis, NATL ACAD SCI LETT, 29, 299-309 (2006).

[9] Srivastava, Pankaj and Sharma, Neeraja. Soft Computing Criterion for ECG Beats Classification and Cardiac Analysis (2012) [Communicated].

[10] Srivastava, Pankaj, Sharma, Neeraja and Singh, Richa. Soft Computing Diagnostic System for Diabetes, International Journal of Computer Applications, 47, 22-27 (2012).

[11] Srivastava, Pankaj and Srivastava, Amit. A note on Soft Computing Approach for Cardiac Analysis, J. Basic. Appl. Sci. Res., 2, 376-385 (2012).

[12] Srivastava, Pankaj and Srivastava, Amit, Spectrum of Soft Computing Risk Assessment Scheme for Hypertension, International Journal of Computer Applications (09758887), 44, 23-30 (2012).

[13] Srivastava, Pankaj, Srivastava, Amit and Sirohi, Ritu. Soft Computing Tools and Classification Criterion for Hepatitis $\mathrm{B}$, International Journal of Research and Reviews in Soft \& Intelligent Computing, 2, 147-153 (2012).

[14] Verma, Priyanka.A Soft Computing Approach for Classification Scheme of ECG Beats, M.Sc. Thesis Environment Science Allahabad University, (2009).

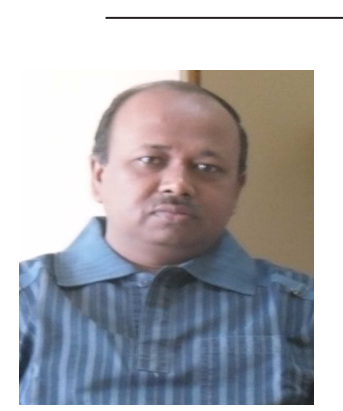

Pankaj Srivastava was born in Allahabad in 1966. He received B.Sc. and M.Sc. degree from Gorakhpur University and Ph.D. degree from Purvanchal University from Jaunpur. For the past thirteen years Prof. Srivastava is with the Department of Mathematics, Motilal Nehru National Institute of Technology Allahabad, India. Currently $\mathrm{He}$ is also serving as Secretary, The National Academy of Sciences India Allahabad (Allahabad Chapter) since January 2011. His research interest includes Application of Soft Computing Techniques in Medical Sciences, Biomathematical Modeling, Tensor Analysis and Ramanujans Mathematics. 


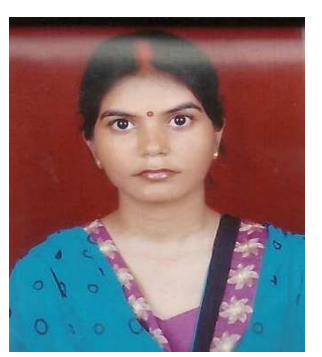

Neeraja Sharma was

born in Allahabad in 1988.

She received B.Sc. degree

from Allahabad University,

Allahabad and M.Sc. degree

from Motilal Nehru National

Institute of Technology

Allahabad. Currently,

she is pursuing $\mathrm{Ph}$. D. in

Soft Computing from Motilal

Nehru National Institute of Technology Allahabad. Her

research interest mainly concerns with Soft Computing and Medical diagnosis. 